\title{
A software agent model of muscle myosin nanomotor
}

\begin{abstract}
The state-of-the-art in information and robotic systems deals with analyzing of natural systems at nanoscale to apply them for constructing potential bio-nanosystems. This paper employs agent technology and introduces a software agent model of muscle myosin nanomotor which illustrates a set of information processes which are running during the mechanism of the nanomotor. Muscle myosin, as a desired dynamic component of potential bio-nanorobotic systems, is the driven motor of muscle contractions. In this work, firstly, muscle myosin nanomotor was introduced as a physical intelligent agent. Then, we have designed the internal decision-making process of the nanomotor using subsumption architecture of agent technology. The agent-based architectural model of the nanomotor was proposed with mapping the subsumption rules of the nanomotor to its respective Deterministic Finite Automaton (DFA). The proposed agent-based architectural DFA model of muscle myosin nanomotor demonstrated that the nanomotor could receive inputs from its environment, analyze data, and generate outputs. Also, the proposed agent-based architectural DFA model of muscle myosin nanomotor was in good agreement with the behavior of the nanomotor inside the muscle cells. Finally, the proposed agent-based architectural DFA model was implemented as a software agent model of the nanomotor. The developed software agent model of muscle myosin nanomotor traced the real behavior of the nanomotor in nature.
\end{abstract}

Keyword: Information process; Information system; Muscle myosin nanomotor; Robotic system; Software agent 